\title{
Parameters Setting of Power System Stabilizer PSS2B
}

\author{
Fan Mengjing ${ }^{1, a}$, Wang Kewen ${ }^{2, b}$, Zhang Jianfen ${ }^{3, c}$ \\ ${ }^{1}$ School of Electrical Engineering, Zhengzhou University, Zhengzhou, China \\ ${ }^{2}$ School of Electrical Engineering, Zhengzhou University, Zhengzhou, China \\ ${ }^{3}$ College of Electric and Information, Jinan University, Zhuhai , China \\ a1664289126@qq.com, bkwwang@ZZU.edu.ca, czhangjianfen@163.com
}

Key words: PSS2B; the shaft torsion; low frequency oscillation; the frequency analysis method; the root locus method; the parameters setting

Abstract. PSS2B is a kind of dual-input power system stabilizer which takes rotational speed deviation and electrical power deviation as its inputs. The shaft torsion signal introduced by the rotational speed deviation have an effect on the low frequency signal each other. The interactions affect the parameters setting of PSS2B. The frequency response analysis method and the root locus method are applied to analyze the interaction. On this basis, the parameters of PSS2B are adjusted. Analyze on an actual generator model.

\section{Introduction}

PSS applied to suppress low-frequency oscillation in power system ${ }^{[1,2,3]}$. IEEE-PSS2A power system stabilizer takes single signal as its input, and PSS2B uses rotational speed deviation and electrical power deviation as its dual-input signal, which can avoid the reverse control caused by the input of single signal and has a good prospect ${ }^{[4,5]}$.

The research of PSS2B power system stabilizer has made some progress. Literature [6] involves field test and parameters setting of PSS2B.In the reference [7], the pre-phase of the PSS2B is reversed to avoid high-angle of lead-ahead compensation. In [8], the parameters setting of PSS with the dual-input signal is converted to the problem about PSS with single input signal by adding the virtual ideal differential module on the side of generator electromagnetic power deviation signal. And the actual grid is verified by digital simulation. Literature [9] has set the parameters of PSS with the a/p-type simulation software. In [10], consider the parameters optimization of PSS2B when the parameters of the mathematical model of the power system are uncertain. More literatures on the PSS2B power system stabilizer have been studied and analyzed. There is no detailed analysis about the problem of shaft torsional vibration when the speed signal is input, including the interaction between torsional vibration and low frequency oscillation and the influence on the parameters tuning of PSS.

In this paper, the interaction between the shaft torsional oscillation and the low frequency oscillation and the influence on the parameters adjustment are discussed. The parameters of PSS2B power system stabilizer are adjusted by phase compensation method and the root locus method, so as to improve the damping capacity of PSS2B.

\section{Construction of Power System Stabilizer PSS2B}

Power system stabilizer PSS2B corresponds to the iv PSS in PSASP (Power System Analysis Software Package) developed by CEPRI, which shown in Fig. $1^{[4]}$. As can be seen from the figure, 
PSS2B takes the speed deviation signal and power deviation signal as the input signals, and sets up the RTF module to reduce or eliminate the impact of the rotor torsional vibration filter when the speed signal is inputted ${ }^{[11]}$.

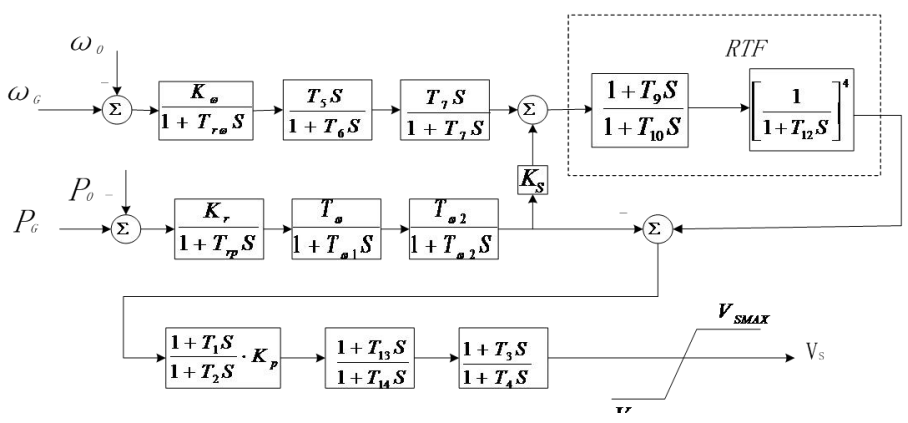

Fig. 1 Construction of the PSS2B

The description of the parameters of PSS2B is as follows: $K_{\omega}$ is the speed deviation magnification ratio, $T_{r \omega}$ is the time constant of speed deviation measurement module, The time constants of the blocking module of the speed signal channel are $T_{5} 、 T_{6} 、 T_{7}$, the time constants of RTF are $T_{9} 、 T_{10} 、 T_{12}, K_{r} 、 K_{s}$ are the power deviation magnification and power matching coefficient. $T_{r p}$ is the time constant of the power deviation measurement module, $T_{\omega} 、 T_{\omega 1} 、 T_{\omega 2}$ are the time constants of the blocking module of the power signal channel, $K_{p}$ is the magnification ratio of PSS, $T_{1} 、 T_{3} 、 T_{13} 、 T_{2} 、 T_{4} 、 T_{14}$ are the lead-lag time constants of PSS.

\section{Impact Analysis of RTF}

By phase compensation, PSS provides positive damping for the system in low frequency band and the sub-synchronous frequency band, and the phase frequency characteristic reflects the effectiveness of damping ${ }^{[12.13]}$. By analyzing the phase frequency characteristics of PSS, the influence of RTF on sub-synchronous signal and low-frequency signal and the interaction between sub-synchronous signal and low-frequency signal are available.

Analysis of the Influence of the Phase - Frequency Characteristics of PSS.When PSS takes speed deviation signal as its input, it will bring the issues about noise and shaft torsional vibration. The main role of RTF is to filter out the torsional vibration signal form the speed deviation signal, and the frequency of torsional vibration signal is located in the sub-synchronous frequency band. Based on the data obtained in the calculation example of the fifth chapter in this paper, the following figures are analysis and explanation. RTF will have a certain impact on the PSS phase-frequency characteristics in the sub-synchronous frequency band, as shown in Fig. 2. 


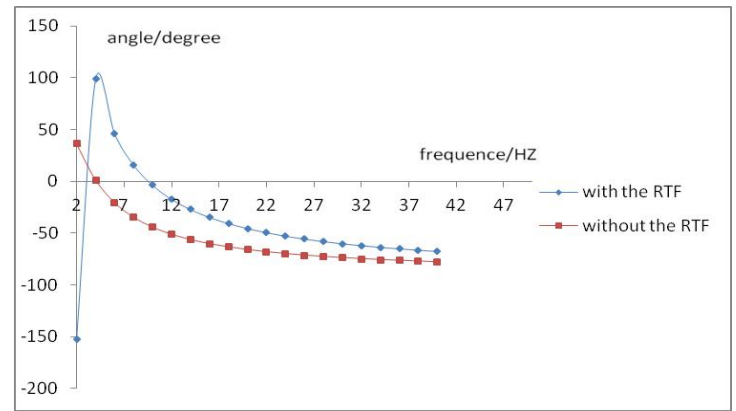

Fig. 2 Influence of RTF in the sub-synchronous frequency

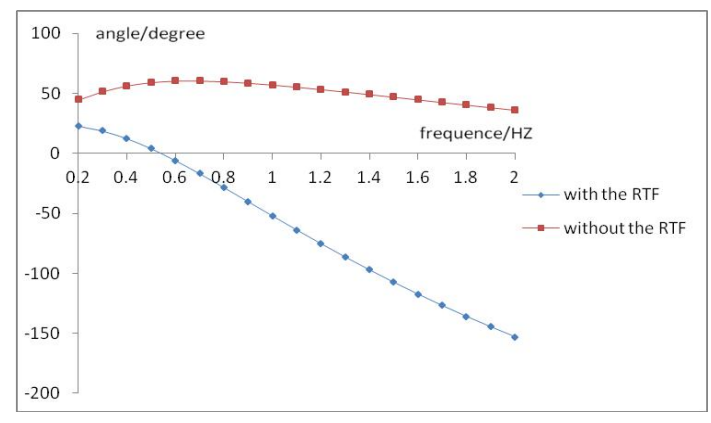

Fig. 3 The influence of RTF in the low frequency

As can be seen from Figure 2, the RTF mainly affects the lower frequency band (2HE 20HZ) of the sub-synchronous frequency band, and has little effect on the higher frequency of the sub-synchronization. Therefore, with the unit capacity increases, the impact of RTF is growing. At the same time, the RTF also affects on low-frequency signal, as shown in Figure 3.

The figure 3 shows that the RTF has a relatively large impact on the low-frequency characteristics. Therefore, the characteristics of sub-synchronous frequency band and low frequency band must be considered when setting the parameters of the RTF.

Analysis of the Effect of $K_{s}$ on Sub-Synchronous Signal and Low Frequency Signal.From the structure of PSS2B, it can be seen that the power matching coefficient $K_{s}$ connects the power signal channel and the RTF. By adjusting $K_{s}$, the participation degree of the power signal can be changed when it passes through the RTF, thus the interaction sub-synchronous signal and low frequency signal is available. As shown in Figure 4, 5.

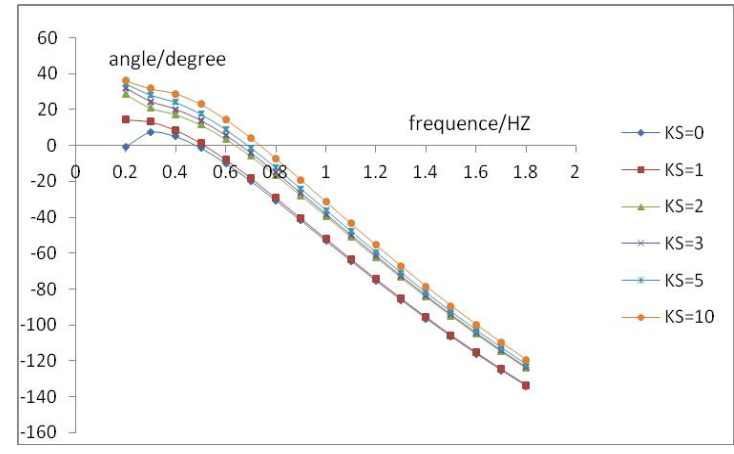

Fig. 4 Coupled effect of low frequency

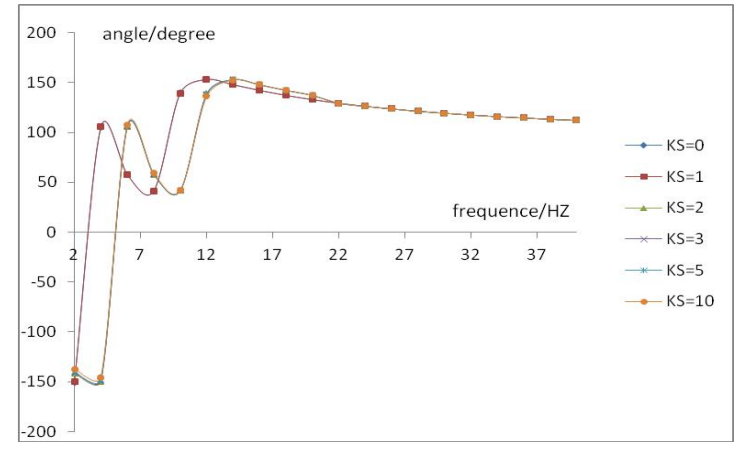

Fig. 5 Coupled effect of sub-synchronous frequency

By changing the value of the $K_{s}$, the different of phase-frequency characteristics of PSS in the entire frequency range is obtained. It can be seen that the compensation characteristic of PSS has a certain change in the lower frequency range; however, in the higher frequency range, it has less variety on its compensation characteristic.

From the above we can know the RTF affects both the low-frequency characteristics and sub-synchronization characteristics, and There is mutual influence between sub-synchronous and low-frequency signals. The parameters setting of the RTF and other parameters adjustment of PSS2B are affected. So in the process of setting parameters, considering synthetically the characteristics of the sub-synchronous band and the low-frequency band is needed. So the system can get better characteristic in entire frequency range 


\section{Phase Lagging Characteristic of Excitation System}

According to the phase lagging characteristic of excitation system, the phase compensation part of PSS is designed, which can provide damping of low frequency oscillation. Based on the same principle, if the phase compensating part of RTF is compensated in the sub-synchronous frequency band to reduce the torsional vibration, so the positive damping is provided in the sub-synchronous frequency band ${ }^{[14]}$.Thus, the damping characteristic of PSS2B will be improved.

The phase lagging characteristic of the excitation system in the sub-synchronous frequency band is required before the phase compensation of the RTF. Since the influence of the low-frequency oscillation, the phase lagging characteristic of entire frequency is necessary, as shown in figure 6.

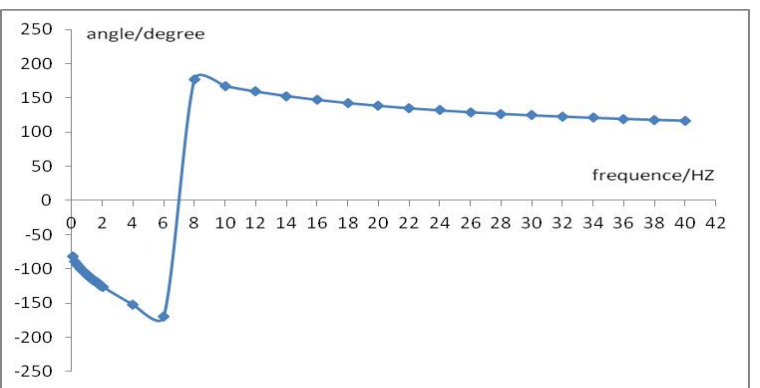

Fig. 6 Phase lags of the excitation system at entire frequency

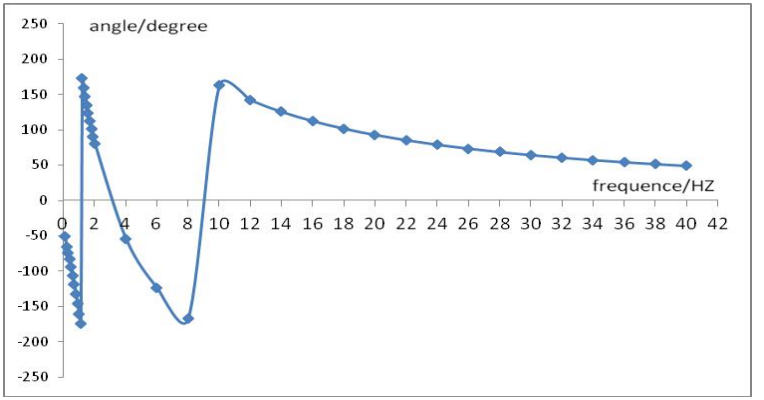

Fig. 7 Compensation characteristic of PSS at entire frequency

The Phase of PSS to be Compensated and the Phase of System after Compensation. Because the signal of PSS involves $-\Delta_{e}$, it leads $\Delta \omega$ about $90^{\circ}$, according to the phase relation, it is necessary to make the compensated electromagnetic torque close to $\Delta \omega$, in order to guarantee the system obtains the positive damping torque. The phase is usually within the range between leading $\Delta \omega$ about $10^{\circ}$ and lagging $\Delta \omega$ about $45^{\circ}$, so it needs to lag $-\Delta_{e}$ within the range between $80^{\circ}$ and $135^{\circ}$. The phase to be compensated of PSS is shown in Figure 7.

Analysis Time Constants of the RTF. According to the lags characteristics of the excitation system at the sub-synchronous frequency band and the characteristics to be compensated of PSS2B at the sub-synchronous frequency band, the time constants of the RTF are set, and that is $T_{9}, T_{10}, T_{12}$.

Now $T_{9}=0.2, T_{10}=0.1$ are fixed, respectively, the value of $T_{12}$ is $0.01,0.02,0.05,0.1,0.2,0.5,1.0$, 2.0, 5.0, 10.0. The frequency characteristic curve is shown in Figure 8.

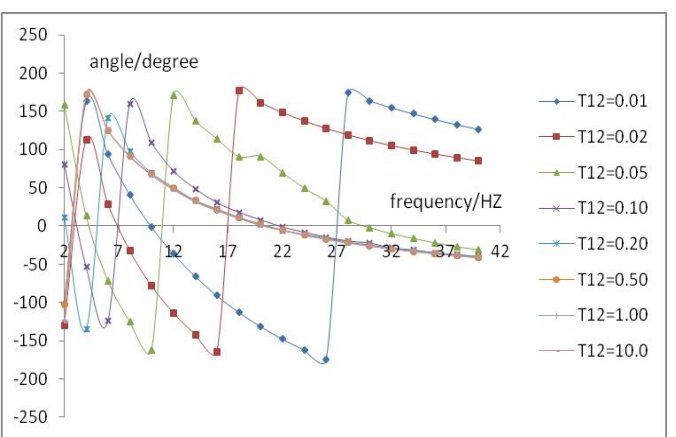

(a) Phase frequency characteristics

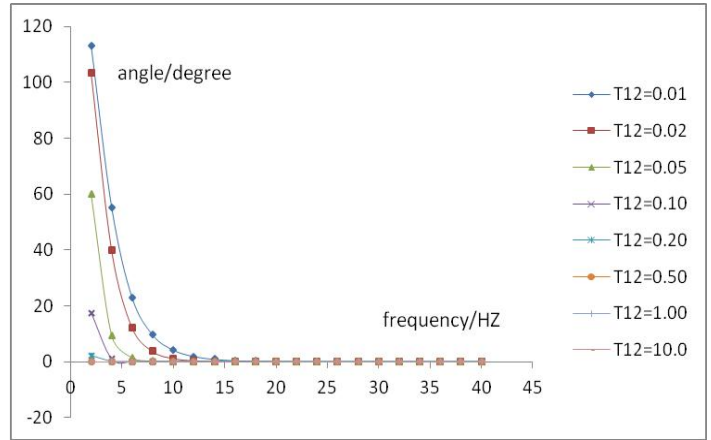

(b)Amplitude frequency characteristics

Fig. 8 Frequency characteristics of the excitation system

It can be seen from the figure a, the phase is close to zero rapidly when $T_{12}$ equals $0.01,0.02$, 
$0.05,0.10$ respectively. And when $T_{12}$ is above 0.10 , the phases at the sub-synchronous frequency band are almost unchanged. The closer to 0 phase in the sub-synchronous frequency, the less impact of the low frequency, so the phase frequency characteristics are obvious. It can be seen from figure $\mathrm{b}$ when $T_{12}$ equals $0.01,0.02,0.05,0.10$ respectively, the amplitudes close to 0 quickly, and when the value of $T_{12}$ is above 0.2 , the magnitude of the amplitude is essentially equal to 0 , which can suppress or eliminate the influence of torsional vibration in the sub-synchronous frequency band. Through the adjustment of the time constants, a good amplitude frequency characteristics is shown at the sub-synchronous frequency band, but the amplitudes of the low-frequency band is affected at the same time.

According the analysis of the time constants of the RTF with the sub-synchronous signal and the low-frequency signal, it can provide some reference for its parameters setting.

\section{Analysis of Gains of PSS2B}

Not only the change of time constants will have an impact on the sub-synchronous signal and low-frequency signal, but also the gain of PSS. In order to analyze the influence of gain on sub-synchronous mode and low-frequency mode, eigenvalue method is applied in this paper. Eigenvalue method is one of the common methods for small signal stability analysis, and the root locus method which based on eigenvalue method is one of the common methods to determine the gain. For the eigenvalue analysis, a linear model of the multi-machine system is established, the eigenvalues are calculated and the correlation factors are analyzed.

Effects of Gains of PSS2B on Sub-Synchronous Mode and Low-Frequency Mode.In the process of gains tuning, it affects the low-frequency oscillation mode, but also affects the sub-synchronization mode due to the RTF. According to the data in the fifth section of this paper, the data are used to analyze and explain the influence of gains which shown in Table1and2.

Table 1 Influence of $K_{p}$ of two modes

\begin{tabular}{ccccc}
\hline gain & \multicolumn{2}{c}{ sub-synchronous } & \multicolumn{2}{c}{ low-frequency } \\
$K_{\omega}$ & $\sigma$ & $\omega$ & $\sigma$ & $\omega$ \\
\hline 0.1 & -0.25727404 & 24.977673 & -0.69530657 & 5.974818 \\
0.3 & -0.46214857 & 25.183967 & -1.13832805 & 5.664977 \\
0.5 & -0.66900675 & 25.510240 & -1.36466285 & 5.286969 \\
0.7 & -0.78918751 & 25.977363 & -2.73176235 & 7.304875 \\
0.9 & -0.77787291 & 26.240959 & -2.56300679 & 7.596660 \\
1.1 & -0.64641038 & 26.716624 & -2.38737748 & 7.960410 \\
2.0 & 1.18099552 & 21.913368 & -2.33216358 & 8.091128 \\
\hline
\end{tabular}

Table 2 Influence of $K_{\omega}$ of two modes

\begin{tabular}{ccccc}
\hline gain & \multicolumn{2}{c}{ sub-synchronous } & \multicolumn{2}{c}{ low-frequency } \\
$K_{\omega}$ & $\sigma$ & $\omega$ & $\sigma$ & $\omega$ \\
\hline 1.0 & -0.27972563 & 26.377990 & -0.38450047 & 4.862266 \\
2.0 & -0.54318319 & 26.597062 & -1.19732722 & 4.508462 \\
2.5 & -0.64641038 & 26.716624 & -2.56300679 & 7.596604 \\
3.0 & -0.73337837 & 26.836287 & -2.07100337 & 8.146401 \\
5.0 & -0.96664245 & 27.289847 & -0.82794415 & 9.399959 \\
6.0 & -1.03363042 & 27.495425 & -0.50965482 & 9.839841 \\
10.0 & -1.12479941 & 28.163734 & 0.54424428 & 10.970120 \\
\hline
\end{tabular}

As can be seen from table 1 , when $K_{p}$ is increasing from 0.1 , the absolute value of the real part of the sub-synchronous mode increases gradually. At $K_{p}$ equals 0.7 , the absolute value of the real part of the sub-synchronous pattern reaches its maximum value, and then decreases gradually. The low frequency mode has the same rule as the change. It can be seen that $K_{p}$ as the amplification 
factor of the PSS has approximately same effect on sub-synchronous mode and low-frequency mode.

However, for the speed deviation amplification factor $K_{\omega}$, it can be seen from Table. 2 that the absolute value of the real part of the eigenvalue of the sub-synchronous pattern increases with the $K_{\omega}$ increasing, while for the low-frequency mode, the absolute value of the real part of the eigenvalue reaches the maximum when $K_{\omega}$ equals 2.5 , then it decreases gradually, and even the real part of the eigenvalue appears positive when $K_{\omega}$ equals 10 . It can be seen that the influence of speed deviation amplification factor $K_{\omega}$ on sub-synchronous and low-frequency modes is different, which has a more direct influence on the sub-synchronous mode introduced with the speed deviation signal.

Thus, the gains affects both the sub-synchronous mode and low-frequency mode, and the influences from different channel gains are may not the same for the two modes. When determining the gain by the root locus method, the analysis of the gain on the sub-synchronous mode and the low-frequency mode can be used to provide some references.

\section{Analysis of Examples}

Experimental System.The system uses 112 generators, of which, 45 generators equipped with PSS2B power system stabilizer. The number of the system nodes is 1126 , and the generator adopts the sixth-order model. The number of the eigenvalue is 3777. Yu Duobao Mountion $2 \mathrm{G}$ is the capacity of $600 \mathrm{MW}$ of the unit. This paper takes the generator as an example to illustrate the process of parameters tuning of PSS2B.

Time Constant Setting. According to the phase lags of the excitation system, we can get the compensation angle of PSS for each frequency, which shown in Table 3.

\begin{tabular}{llll}
\multicolumn{4}{c}{ Table 3 Needed compensation phases of PSS2B } \\
\hline Low & compensation & sub- & compensation \\
frequency & phase & synchronous & phase \\
\hline 0.10 & 31.0934 & 2.70 & 141.6996 \\
0.21 & 23.2827 & 3.90 & 98.5252 \\
0.30 & 18.8503 & 6.80 & 29.1193 \\
0.65 & -5.7991 & 9.00 & 5.0621 \\
0.80 & -28.3297 & 11.3 & -3.7453 \\
1.10 & -63.7023 & 13.0 & -22.6465 \\
1.20 & -75.1542 & 15.9 & -34.8438 \\
1.53 & -107.3645 & 18.0 & -40.8102 \\
\hline
\end{tabular}

\begin{tabular}{|c|c|c|c|c|}
\hline $\begin{array}{c}\text { time } \\
\text { constants }\end{array}$ & $\begin{array}{l}\text { setting } \\
\mathrm{s}\end{array}$ & $\begin{array}{l}\text { time } \\
\text { constants }\end{array}$ & $\mathrm{g}$ & settin \\
\hline $\mathrm{T}_{1}$ & 0.50 & $\mathrm{~T}_{14}$ & & 0.10 \\
\hline $\mathrm{T}_{2}$ & 0.05 & $\mathrm{~T}_{9}$ & & 0.20 \\
\hline $\mathrm{T}_{\mathrm{a}}$ & 0.30 & $\mathrm{~T}_{10}$ & & 0.10 \\
\hline $\mathrm{T}_{4}$ & 0.03 & $\mathrm{~T}_{12}$ & & 0.10 \\
\hline
\end{tabular}

The time constants of lead-lag module are set by the above method combining with the influences of the time constants on the sub-synchronous signal and low-frequency signal analysis. Time constants are shown in Table 4.

Determine the gain of the PSS2B. Using the root locus method, the effect of stability on the low frequency oscillation mode by changing the value of gains is investigated. Thereby the optimal 
value of the gain is available. Symbol $\sigma$ represents the real part of the eigenvalue, and the low-frequency oscillation mode uses symbol $\omega$ to represent it. From the root locus curve, it can be observed more clearly and intuitively that the change of eigenvalue with the $K_{\omega}$ changing. The curve is shown in Fig. 10.

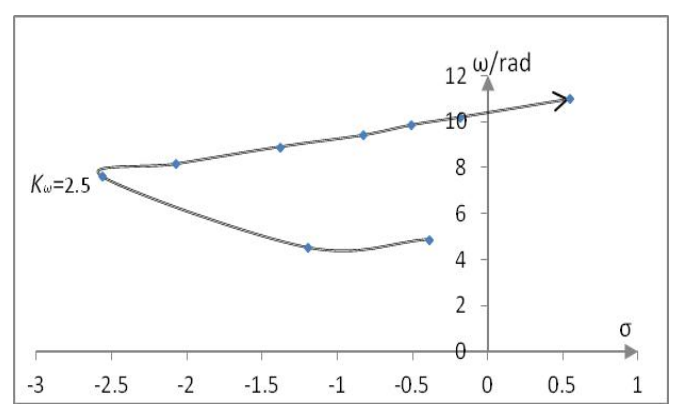

Fig. 9 Root locus with the change of $K_{\omega}$

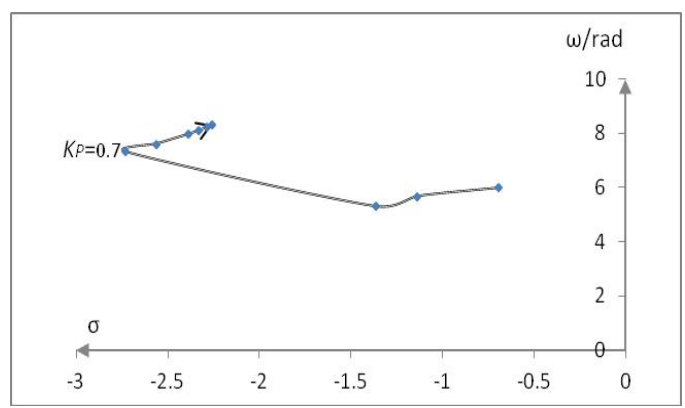

Fig. 10 Root locus with the change of $K_{P}$

It can be easily seen from Fig. 9that the damping torque increases with $K_{\omega}$ increasing. If the value of $K_{\omega}$ is so large that the compensation phase is not appropriate, and the system is unstable gradually, even a positive real part of eigenvalue is present, so the system is instability finally. It can be seen from Figure9that the optimum value of $K_{\omega}$ is about $1 / 4$ of the critical value of $K_{\omega}$, i.e. $K_{\omega}$ is about 2.5 .

The root locus with the change of $K_{P}$ is shown in Fig.10. From Fig.10, the absolute value of the real part of eigenvalue increases with $K_{P}$ increasing, and when $K_{P}$ is about 0.7 , the absolute value of real part reaches its maximum. If it continues to increase, the absolute value began to become small, at the same time the frequency is barely change. Therefore, the root locus curve shows that the value of the optimal gain of $K_{P}$ is about 0.7 . Combining the analysis of gains of sub-synchronous mode and low frequency mode, the value of gains are shown in Table 5.

\begin{tabular}{cc}
\multicolumn{2}{c}{ Table 5 Gains of PSS2B } \\
\hline gains & settings \\
\hline$K_{w}$ & 2.50 \\
$K_{p}$ & 0.70 \\
$K_{y}$ & 0.15 \\
$K_{S}$ & 1.00 \\
\hline
\end{tabular}

\section{Conclusions}

The dual-input power system stabilizer of PSS2B is more and more widely applied with its own characteristics and advantages. This paper analyzes the structure and frequency characteristics of PSS2B. By the phase-frequency characteristic analysis method and the root locus method, the influence of the interaction between the sub-synchronous signal and the low-frequency signal is analyzed, and it has effect on the parameter setting of PSS2B. On this basis, by adjusting the parameters, the damping characteristics of PSS2B can be improved. In this paper, a generator from 
multi-machine system as an example is applied to analyzed and explained.

\section{References}

[1] Yixin Ni, Shousun Chen, Baolin Zhang. Analysis of Dynamic Power System. Tsinghua University Press (2002) (In Chinese).

[2] Sili Fang, Fang Zhu. The Principle and Application of Power System Stabilizer. China Power Press,(1996)( In Chinese).

[3] Fang Zhu, Hongguang Zhao, Zenghuang Liu, et al. Proceedings of the CSEE, 2007, 7(1): 1-7. ( In Chinese).

[4] IEEE Power Engineering Society, IEEE Std 421.5-2005. Recommended practice for power system stability studies [S].2005.

[5] YANG X, FELIACHI. A Stabilization of inter-area oscillation modes through excitation systems[J].IEEE Trans on Power System, 1994, 9(1): 494-502.

[6] Lihuan Yang, Feng Xu, Huarong Hu, et al.. Power System Protection and Control, 2010, 38(1): 112-114, 128. ( In Chinese).

[7] Kuangyu Wu, Cencen Lu, Wu Long, et al. A New PSS With Double-Signal Input and Its Simulation Research[J]. Power System Technology, 2016, 40(5): 1462-1468. (In Chinese).

[8] Yuqiang Hou, Qinfeng Ma, Wei Li, et al. Southern Power System Technology, 2012, 6(2): 89-93. ( In Chinese).

[9] Wei Xue, Dagong Li, Yangfan Ou. Power System Protection and Control, 2012, 40(10): 101-105. ( In Chinese).

[10] Stefan Paszek, Adrian Nocon. Parameter polyoptimization of PSS2A power system stabilizers operating in a multi-machine power system including the uncertainty of model parameters[J]. Applied Mathematics and Computation, 2015, 26(7): 750-757.

[11] Xiaorong Xie, Yingduo Han, Jiuxi Guo. Analysis and Control of Sub-synchronous Resonance: Science Press, 2015.( In Chinese).

[12] Fan Zhang, Zheng Xu. Transactions of China Electro technical Society, 2007, 22(6): 121-127. ( In Chinese)

[13] Ping Jiang, Nan Li. Electric Power Automation Equipment, 2010, 30(7): 40-44. ( In Chinese)

[14] Ping Jiang, Guanyi Wang. Transactions of China Electro technical Society, 2009, 24(10), 122-127. ( In Chinese) 